\title{
Human Urinary Kallidinogenase (HUK) Alleviates Myocardial Injury in Rats with Coronary Microembolism Through PI3K/Akt/Fox01 Signaling Pathway.
}

Jian Xie

Guangxi Medical University First Affiliated Hospital

Yunhua Lin

Guangxi Medical University

Guoqing Liu

Guangxi Medical University

Qingqing Nong

Guangxi Medical University

\section{Bingling Wu}

Guangxi Medical University

\section{Yuqian Xie}

Guangxi Medical University

\section{Tao Li}

Guangxi Medical University First Affiliated Hospital

Lang Li ( $\sim$ drlilang@126.com)

Guangxi Medical University First Affiliated Hospital https://orcid.org/0000-0001-7273-3615

\section{Research Article}

Keywords: Human Urinary Kallidinogenase, Coronary artery microembolization, PI3K/Akt/FoxO1, myocardial inflammation, apoptosis

Posted Date: November 11th, 2021

DOI: https://doi.org/10.21203/rs.3.rs-1054881/v1

License: (9) (1) This work is licensed under a Creative Commons Attribution 4.0 International License. Read Full License 


\section{Abstract}

\section{Purpose}

Coronary artery microembolization (CME) is a severe clinical complication that can cause myocardial infarction, induce myocardial inflammation and apoptosis, ultimately lead to cardiac dysfunction. Human Urinary Kallidinogenase (HUK), a glycoprotein found in human urine, its role and underlying mechanism in CME are still unclear. Therefore, our goal is to explore the effect of HUK on the PI3K/Akt/FoxO1 axis of $\mathrm{CME}$ in rats, determine whether it can restrain myocardial inflammation and apoptosis, and alleviate CME-induced myocardial injury.

\section{Methods}

We split 40 Sprague-Dawley (SD) rats into CME, CME + HUK, CME + HUK + LY294002 (CME + HUK + LY), and sham operation groups randomly (10 animals in each group). The dosage of HUK was 0.016 $\mathrm{PNA} / \mathrm{kg} /$ day. Also, $42 \mu \mathrm{m}$ inert plastic microspheres were injected into the left ventricle of rats to establish the CME model. Notably, rats in the CME+HUK+LY group were injected $10 \mathrm{mg} / \mathrm{kg}$ of LY294002 (a particular inhibitor of $\mathrm{P} I 3 \mathrm{~K}$ ) intraperitoneally 30 minutes before modeling. We measured cardiac function 12 hours after the operation. Besides, the serum of myocardial injury biomarkers and myocardial inflammation, as well as apoptosis-related genes were measured, the myocardial histopathological examination was also performed.

\section{Results}

Our results indicated that $\mathrm{CME}$ induced myocardial inflammation and apoptosis, also caused myocardial infarction. HUK mainly activated PI3K/Akt/FoxO1 signal transduction, effectively reducing myocardial inflammation, apoptosis, and myocardial infarction area and improving CME-induced myocardial injury. In addition, these cardioprotective effects can be reduced by PI3K specific inhibitor LY294002, suggesting that the above protective effects may be related to the process of activation of the PI3K/ Akt /FoxO1 axis.

\section{Conclusion}

Our findings revealed that HUK might restrain myocardial inflammation and apoptosis by activating the $\mathrm{PI3K} / \mathrm{Akt} /$ FoxO1 axis, thus ameliorating CME-induced myocardial injury.

\section{Introduction}

Coronary artery microembolism (CME) is a spontaneous event that rises from atherosclerotic plaque rupture in acute coronary syndrome (ACS) or coronary intervention $[1,2]$. It is considered to be the main complication of percutaneous coronary intervention (PCI) [3]. CME may cause no-reflow of the myocardium, leading to severe cardiac dysfunction and arrhythmia [4]. Besides, a study by Gerd Heusch et al. indicated that repetitive CME could cause progressive loss of functional cardiomyocytes and induce heart failure [5]. It is also reported that the rat $\mathrm{CME}$ has a local myocardial infarction, and there are a large 
number of apoptotic and necrotic cells in the corresponding area [6]. In summary, the existing studies indicate that myocardial inflammation and apoptosis may play a vital part in CME-induced myocardial damage. So, if targeted interventions on these aspects might be critical to the treatment of myocardial protection [7-9].

Recently, the potential protective mechanism of the PI3K/ Akt axis in CME has received extensive attention. For example, previous studies have shown that gustazine can inhibit oxidative stress and inflammation by influencing the PI3K/Akt pathway, thereby reducing myocardial damage caused by CME in rats [10]. Human Urinary Kallidinogenase (HUK), a glycoprotein found in male urine, as a modulator of the kallikrein-kinin system, has been used in a wide range of patients diagnosed with ischemic stroke [11]. Ning Ma et al. confirmed that HUK acts as the factor of anti-inflammatory and anti-apoptotic through $\mathrm{PI} 3 \mathrm{~K} / \mathrm{Akt} / \mathrm{FoxO} 1$ signaling pathway, thereby reducing brain damage in rats with persisting middle cerebral artery occlusion [12]. However, whether HUK can reduce myocardial damage and improve cardiac function caused by CME has not been fully elucidated, and further research is still needed.

Hence, our study aims to explore the effect of HUK on myocardial inflammation and apoptosis and explore its role in the $\mathrm{PI} 3 \mathrm{~K} / \mathrm{Akt} / \mathrm{Fox} 01$ pathway to determine whether it is related to reducing myocardial damage caused by CME and improving cardiac function.

\section{Materials And Methods}

\section{Grouping and CME model establishment}

All experimental animals were procured from the Experimental Animal Center of Guangxi Medical University. The Animal Ethics Committee has approved all experimental protocols and procedures of Guangxi Medical University. First, altogether 40 male adult Sprague-Dawley (SD) rats weighing 250-300g were divided into $\mathrm{CME}, \mathrm{CME}+\mathrm{HUK}$ treatment, $\mathrm{CME}+\mathrm{HUK}+\mathrm{LY}$, and sham operation group (Sham group) randomly (ten in each group). Subsequently, we constructed the CME model according to the methods reported in previous studies [9]. Briefly, the intraperitoneal injection was performed (pentobarbital sodium, $30-40 \mathrm{mg} / \mathrm{kg}$ ) to anesthetizes the SD rats. We used a small animal ventilator to assist breathing. We conducted a left-sided thoracotomy in the position of the incision between the third and fourth ribs of the rat. The ascending aorta was separated and gripped for 10s. At the same time, we injected $300042 \mu \mathrm{m}$ plastic microspheres (BioSphere Medical Inc., USA) suspended in $0.1 \mathrm{~mL}$ sodium lauryl sulfate into the left ventricle through the cardiac apex. As for the Sham group, an equal volume of saline was injected into the left ventricle in the same way. The CME+HUK group and CME+HUK+LY group were given HUK $0.016 \mathrm{PNA} / \mathrm{kg} / \mathrm{day}$ through tail vein injection for 7 consecutive days before the operation. Before the operation, The CME+HUK+LY group was injected $10 \mathrm{mg} / \mathrm{kg}$ of LY294002 (a particular inhibitor of PI3K) (APExBIO, Houston, USA) intraperitoneally 30 minutes. All animals were injected with penicillin to prevent infection.

\section{Cardiac-function measurements}


According to a recent study, the cardiac function of rats is at the lowest level after $12 \mathrm{~h}$ of the modeling operation[4]. At this time point, in order to evaluate the cardiac function of the rat, we used the Philips Sonos7500 system (Philips Technologies, USA) to measure the left ventricular fraction shortening (LVFS), left ventricular ejection fraction (LVEF), left ventricular end-systolic diameter (LVESd) and left ventricular end-diastolic diameter (LVEDd) respectively. The measurement was repeated 3 times for each rat, and the average value was taken. The above operations were all done using the $\mathrm{S} 12$ probe with a $12 \mathrm{MHz}$ probe. Experienced cardiac sonographers were used to carry out all ultrasound examinations.

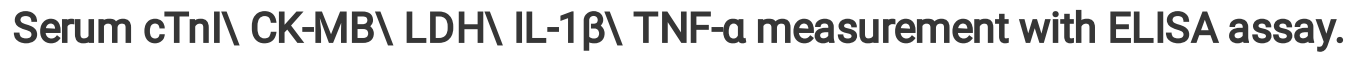

Blood samples were drawn from the abdominal aorta before sacrificing the rats. Then, the levels of serum cardiac troponin I (cTnl), creatine kinase myocardial band isoenzyme (CK-MB), lactate dehydrogenase (LDH), IL-1 $\beta$, and TNF- $a$ were estimated by utilizing an Enzyme-linked immunosorbent assay (ELISA) based on the instructions from the manufacturer. All the above tests were performed using standardized ELISA kits (Bio-Swamp Biological Technology Co., Ltd, China)

\section{Sample collection and processing}

Twelve hours after the operation, the rats were sacrificed for sampling after the ultrasound examination was completed. We performed intraperitoneal injection (pentobarbital sodium, $60 \mathrm{mg} / \mathrm{kg}$ ) to anesthetize the rats. After rats' blood was collected from the abdominal aorta, subsequently, the heart was isolated shortly after opening the chest. Then the auricle and atrium were excised, and the cardiac tissue was separated into upper, mid, as well as bottom sections. The upper and mid was transferred to a refrigerator at $-80^{\circ} \mathrm{C}$ to prepare for real-time quantitative PCR (RT-qPCR) as well as Western blotting. $4 \%$ paraformaldehyde was used to fix the bottom section for 12 hours. Then, it was embedded with paraffin and later used for slice preparation. After hematoxylin-eosin (H\&E) staining, the condition of cardiomyocytes after CME was observed via an optical microscope. Hematoxylin-basic fuchsin-picric acid (HBFP) staining was used to identify myocardial ischemia, and TdT-mediated dUTP nick end labeling (TUNEL) staining was utilized to identify the apoptosis of cardiomyocyte.

\section{Detection of myocardial microinfarction area}

We detected myocardial ischemia via HBFP staining, which enables ischemic cardiomyocytes to turn red and staining the cytoplasm as well as the nucleus of healthy cardiomyocytes yellow and blue. Besides, we observed every stained slice with the aid of a DMR + Q550 pathology image analyzer (Leica, Wetzlar, Germany). We obtained five fields of view from every slice randomly. Leica Qwin software, as well as the planar method, were applied to calculate the infarct area. Next, the infarct area was divided by the whole observation size to measure the infarct percentage.

\section{Detection of cardiomyocyte apoptosis}

TUNEL assay (Roche, USA) was performed utilizing a TUNEL apoptosis detection kit to detect cardiomyocyte apoptosis based on the manufacturer's instructions. The apoptotic cell nucleus was 
yellow-brown under the observation of an optical microscope. 40 non-overlapping regions were randomly selected from each slice ( $\times 400$ magnification). Then, the total amount of apoptotic cardiomyocytes, as well as cardiomyocytes, were counted. The rate of apoptotic cardiomyocytes was obtained with the formula of the count of apoptotic cardiomyocytes/ the gross count of cardiomyocytes.

\section{Immunofluorescence (IF) Staining}

We prepared slices with a thickness of $4 \mu \mathrm{m}$ and performed immunofluorescence staining based on the instructions provided by the manufacturer. After completing the previous step, the slices were incubated with the three antibodies (cleaved caspase-3 (\#9661, Cell Signaling Technologies, Danvers, MA, USA), Bax (ab53154, Abcam, Cambridge, UK), and Bcl-2 (ab194583, Abcam) at $4^{\circ} \mathrm{C}$ all-night. PBS was utilized to rinsed slices five times after incubation with primary antibodies. Then, slices were incubated with fluorescent secondary antibodies for $50 \mathrm{~min}$ under the temperature of the room. Subsequently, a fluorescence microscope (Olympus, Tokyo, Japan) was used to acquire the images after using the 4',6diamidino-2-phenylindole (DAPI) to counterstain the nuclei for 7 minutes.

\section{Real-time quantitative PCR quantitative measurement}

A tissue sample (200 mg) of cardiac tissues was obtained for real-time quantitative PCR (RT-qPCR). According to the directives of the kit, we extracted the total RNA of inflammation-related genes (IL-1 $\beta$, TNF-a) and used RT-qPCR to identify their relative expression levels in CME. Furthermore, we also measured the relative expression of $\mathrm{Bax}, \mathrm{Bcl}-2$, and caspase-3, which were associated with apoptosis in the same way. The ABI PRISM-7500 machine (CA, USA) was used to perform RT-qPCR using the TB Green ${ }^{\circledR}$ Premix Ex Taq $^{\text {TM }}$ II (TaKaRa, Japan). We listed the specific primer sequences adopted in this experiment in Table 1.

\section{Western blotting analysis}

Sodium lauryl sulfate-polyacrylamide gel electrophoresis $(10 \% \sim 15 \%)$ was utilized to split the total protein obtained from cardiac tissues and then transferred it to a polyvinylidene fluoride membrane (PVDF, Millipore, Atlanta, Georgia, United States). Subsequently, 5\% bovine serum albumin (BSA) was used for sealing the membrane for 1 hour under room temperature. Corresponding primary antibodies allnight (PI3K (ab40776), p-PI3K (Tyr607) (ab182651), Akt (\#9272), p-Akt (Ser473) (ab9271), Fox01 (ab179450), p-FoxO1 (ab259337), Bax (ab53154), Bcl-2 (ab194583), GAPDH (\#5174), and cleaved caspase-3 (\#9661)) were then incubated at $4^{\circ} \mathrm{C}$. After completing the above processing, the membrane was washed with Tris-buffered saline including $0.1 \%$ Tween 20 (TBST) five times. Then, we incubated the secondary antibody (ab6721, 1:10000, Abcam) coupled with horseradish peroxidase in TBST for 2 hours at room temperature. All antibodies (1:1000 dilution) used in our study were procured from Cell Signaling Technology (Beverly, MA, USA). An enhanced chemiluminescence detection system (Pierce, Rockford, IL, USA) was used to test the signals. The densitometry technique was used to quantify the protein bands via Image J software ( $\mathrm{NIH}$, USA). 


\section{Statistical Analysis}

SPSS software (version 23.0, IBM, Chicago, USA) was used to perform statistical data analysis. Obtained experimental data were shown as mean \pm SD (standard deviation). Use One-way Analysis of Variance (ANOVA) to determine the variance of the difference. GraphPad Prism software was applied for all statistical tests. The statistical difference is regarded as significant when $\mathrm{P}<0.05$.

\section{Result}

\section{Effect of HUK on Cardiac Function of SD Rats.}

We measured LVEF, LVFS, LVEDd, and LVESd 12 hours after successful CME modeling to evaluate the cardiac function of rats. Fig. 1 showed the echocardiogram results of each group. Our study indicated that compared with the Sham group, the values of LVEF and LVFS in the CME group decreased.

Meanwhile, the LVEDd and LVESd increased (all P <0.05), indicating that the myocardial contractility and left ventricular enlargement of the CME group were significantly impaired. In addition, the CME+HUK group had an improved cardiac function, higher LVEF, and LVFS, as well as lower LVEDd and LVESd than the CME group (all P <0.05). What's more, LY294002 counteracted the cardiac function improved by HUK.

\section{Effect of HUK on the microinfarct size after CME.}

H\&E and HBFP staining showed only a few sporadic ischemic areas in the Sham group, and no infarction occurred in most areas (Fig. 2A and 2B). In CME, CME+HUK, and CME+HUK+LY groups, micro-infarct areas could be seen, most of the infarct areas were located in the left ventricle, and embolized microspheres can be seen in the field of vision. The results of H\&E staining indicated that cardiomyocytes of the micro-infarct area were of no nucleus or ruptured nucleus; the cytoplasm was also red. In addition, the area around the infarct showed swelling and degeneration of cardiomyocytes, red blood cell effusion, as well as inflammatory cell infiltration simultaneously. Our HBFP staining results showed that the infarct area of the CME rat was significantly wider than that in the Sham group $(P<0.05)$. Besides, the infarct area in the CME rat was also significantly wider than that in the CME+HUK group $(P<0.05)$. Our results indicated that HUK pretreatment might reduce the myocardial microinfarction area, which was inducted by CME in rats. Whereas the treatment of combining LY294002 and HUK reduced the effect of HUK on the myocardial microinfarct area.

\section{Effect of HUK on cardiomyocyte apoptosis in rats via various methods.}

TUNEL staining results (Fig. 3) showed that the infarcted and normal physiology cardiomyocyte nuclei were stained yellow and light blue, respectively. Apoptotic cardiomyocytes are mainly distributed in the area around myocardial microinfarction. The apoptosis rate of cardiomyocytes in the CME group was higher than that in the Sham and CME+HUK groups significantly (both $\mathrm{P}<0.05$ ), and the therapy with an alliance of LY294002 and HUK balanced out the anti-apoptotic function of HUK. Besides, we measured the expression levels of apoptosis-associated genes from the mRNA and protein by RT-qPCR as well as 
Western blotting. RT-qPCR analysis (Fig. 4A) results showed that the expression levels of caspase-3 and Bax in the myocardial tissue of the CME group increased than the Sham and CME+HUK groups significantly (both $\mathrm{P}<0.05$ ). On the contrary, the expression level of $\mathrm{Bcl}-2$ in the myocardial tissue of the CME group reduced than the Sham and CME+HUK groups significantly (both $\mathrm{P}<0.05$ ). Besides, the expression levels of Bax and caspase- 3 in cardiomyocytes in the CME+HUK+LY group were statistically significantly higher than that in the CME+HUK group $(P<0.05)$. In contrast, the expression of $B c 1-2$ in the CME group was statistically significantly down-regulated $(P<0.05)$. Western blotting analysis (Fig. 4B) also showed that the relative expression levels of $\mathrm{Bax}, \mathrm{Bcl}-2$, and Cleaved caspase- 3 at the protein level in each group of cardiomyocytes were consistent with the above results.

Further, we also found that Bax and Cleaved caspase-3 expression levels significantly decreased in the Sham and CME+HUK groups than that in the CME and CME+HUK+LY groups based on the results from immunofluorescence staining, and $\mathrm{BCl}-2$ in the $\mathrm{CME}$ and $\mathrm{CME}+\mathrm{HUK}+\mathrm{LY}$ groups were statistically significantly down-regulated (Fig. 5). Summarily, above all results showed that preliminary treatment with HUK could inhibit CME-caused apoptosis in cardiomyocytes effectively. In addition, the effect of HUK can be eliminated by the pretreatment of LY294002.

\section{Effect of HUK on the inflammation of cardiomyocytes.}

To explore the effect of HUK on the cardiomyocyte's inflammation, three methods (Elisa, RT-qPCR, as well as Western blotting analysis) were applied to identify the relative expression levels of IL-1 $\beta$ and TNF-a of the different samples (Fig. 6). Interestingly, we finally obtained a consistent result. Briefly, the measurement results of the above three methods all indicated that the expression levels of IL-1 $\beta$, as well as TNF-a in the CME group, were significantly higher than that of the Sham group. Also, compared with the CME group, the expression levels of IL-1 $\beta$ and TNF- $\alpha$ in the CME+HUK group were statistically significantly lower (both $\mathrm{P}<0.05$ ). The expression levels of IL-1 $\beta$ and TNF- $\mathrm{a}$ of the CME+HUK+LY group were significantly higher than of the $\mathrm{CME}+\mathrm{HUK}$ group $(\mathrm{P}<0.05)$. In short, our study revealed that preliminary treatment with HUK could alleviate CME-caused inflammation in cardiomyocytes efficiently. However, the effect of HUK on reducing TNF- $\alpha$ and IL-1 $\beta$ levels could be eliminated by LY294002.

\section{Serum Ck-mblctniVldh Levels}

In our current study, we measured the serum myocardial injury biomarkers levels (Fig. 7). Briefly, the degree of myocardial damage was assessed by calculating the serum CTnI, LDH, and CK-MB levels. The CME group had statistically significantly higher serum CK-MB, cTnl, and LDH levels than the Sham group. In addition, the serum LDH, cTnl, and CK-MB levels in the CME+HUK group were statistically significantly lower than those in the CME group. Compared with the CME+HUK group, the serum CK-MB, CTnl, and LDH levels were statistically significantly lower in the $C M E+H U K+L Y$ group (all $P<0.05$ ).

\section{Effect of HUK on the PI3K/Akt/FoxO1 signaling pathway.}


Here, we determined the relative expression levels of PI3K/Akt/Fox01 signaling pathway marker genes(pPI3K, PI3K, p-Akt, Akt, p-Fox01, Fox01)from the protein level via the method of Western blotting analysis(Fig. 8). It indicated that the expression levels of p-PI3K, p-Akt, and p-FoxO1 in the CME group were significantly lower than those in the Sham and CME+HUK groups (all $\mathrm{P}<0.05$ ). Compared with the CME+HUK group, the expression levels of p-PI3K, p-Akt, as well as p-FoxO1 in the CME+HUK+LY group were lower significantly (all $\mathrm{P}<0.05$ ). Also, compared with the CME group, the rates of $p-P I 3 K / P I 3 K, p-$ Akt/Akt, as well as p-Fox01/FoxO1 in the Sham and CME+HUK groups were significantly higher (all $\mathrm{P}$ $<0.05)$. In addition, the rates of $\mathrm{p}-\mathrm{PI} 3 \mathrm{~K} / \mathrm{PI} \mathrm{KK}, \mathrm{p}-\mathrm{Akt} / \mathrm{Akt}$ as well as $\mathrm{p}-\mathrm{Fox} 01 / \mathrm{FoxO} 1$ in the CME+HUK+LY group were significantly lower than those in the CME+HUK group $(P<0.05)$. Our results revealed that HUK participates in the process of activating the PI3K/Akt/FoxO1 signaling pathway.

\section{Discussion}

As a common $\mathrm{PCl}$ complication, Coronary artery microembolization (CME) can be seen in acute coronary syndrome (ACS) patients. The incidence of CME during perioperative $\mathrm{PCl}$ is $15 \% \sim 20 \%$, and it is as high as $45 \%$ in patients with high risk. CME may cause postoperative "no flow" or "slow flow" and microcirculation dysfunction, which in turn may lead to myocardial ischemia, arrhythmia, heart failure, and other consequences [13]. Given its high incidence, it has been viewed as an independent predictor of heart disease incidence and mortality [14].

Human Urinary Kallidinogenase (HUK) is the tissue kallikrein extracted from urine. It has been advocated as a national class I new drug for treating stroke patients in China [15]. Existing studies have shown that HUK can reduce the formation of atherosclerosis and restrict intimal hyperplasia by down-regulating TGF$\beta 1$ expression and Smad2/3 phosphorylation, and up-regulating eNOS activity [16]. In addition, previous studies have shown that HUK can effectively inhibit inflammation and prevent ischemic brain damage by activating the MAPK/ERK pathway or down-regulating the NF-KB pathway [17]. Also, the previous study conducted by Han et al. indicated that HUK acted neuroprotective functions, including reduce infarct size and improve neurological deficits via up-regulating VEGF and the apelin/APJ pathway [15]. In short, existing research showed that HUK might have promising research prospects in coronary atherosclerotic heart disease.

The PI3K/Akt signaling pathway is associated with regulate physiological functions such as apoptosis and inflammation. Its activation can reduce myocardial apoptosis and inflammation, thus reducing myocardial damage effectively [18-20]. The previous study indicated that the PI3K/Akt signaling pathway was related to the process of various genes transcription, including rapamycin(mTOR), glycogen synthase kinase 3(GSK-3), forkhead box protein 01/3(Fox01/3), as well as nitric oxide synthase (NOS) et al. [21]. Fan He et al. found that Lipopolysaccharide could reduce I/R-induced brain damage, inhibit nerve cell apoptosis, and protect nerve cells via PI3K/Akt/FoxO1 signaling pathway [22]. Another study also showed that HUK could reduce apoptosis and inflammation in acute ischemic stroke by activating the $\mathrm{PI3K} / \mathrm{AKT} /$ FoxO1 axis, thereby playing a protective role against brain injury [12]. However, the effect of HUK on the PI3K/Akt/FoxO1 axis in CME and its role in CME have not been thoroughly studied. 
Our previous studies have shown that CME usually leads to cardiomyocyte apoptosis and inflammation, which may cause cardiac dysfunction [6,23]. For cardiomyocyte apoptosis, the previous researches showed that the significant cardiomyocyte apoptosis and abnormal expression of apoptosis-related genes (up-regulated Bax and Cleaved caspase-3; down-regulated Bcl-2) in the infarct zone [24, 25]. For cardiomyocyte inflammation, the expression levels of inflammation-related genes (IL-1 $\beta$, TNF-a) in the CME group were statistically significantly up-regulated than those in the Sham group $[26,27]$. The above research results are consistent with ours, which shows that our current experimental results are credible to a certain extent.

Our study found that CME indeed caused inflammation and increased apoptosis in rat cardiomyocytes, thus leading to severe damage to the myocardium. And HUK (pretreatment starting 7 days before CME) is involved in activating the $\mathrm{PI} 3 \mathrm{~K} / \mathrm{Akt} / \mathrm{FoxO} 1$ axis, decreasing myocardial inflammation and apoptosis, thereby exerting a cardioprotective effect in CME. On the contrary, when we treated with PI3K specific inhibitor LY294002, the essential proteins p-PI3K, p-Akt, as well as p-Fox01 were statistically significant down-regulated, indicating that $\mathrm{PI} 3 \mathrm{~K} / \mathrm{Akt} / \mathrm{Fox} 01$ signaling pathway was suppressed. The expression levels of inflammation-related genes (IL-1 $\beta$, TNF-a) and pro-apoptotic genes (Bax, Cleaved caspase-3) increased significantly, while Bcl-2 was decreased. Thus, the cardioprotective effect of HUK was reduced by the application of LY294002

The limitations of our study are as follows. Firstly, the pathophysiological changes of the CME rat model established by using plastic microspheres without biological characteristics (such as thrombosis, vascular activity, etc.) are different from those caused by atherosclerotic plaque in the clinic. Secondly, we investigated the effect of HUK on inhibiting myocardial inflammation and apoptosis induced by CME through the PI3K/Akt/Fox01 signaling pathway. However, we only used PI3K specific inhibitor (LY294002) to affect PI3K/Akt/FoxO1 signaling pathway, it is also necessary to use inhibitors or agonists of PI3K downstream molecules to further and better study the effect of HUK on the PI3K/Akt/FoxO1 signaling pathway. Finally, other signaling pathways are likely to participate, and the influence of other pathways is not ruled out, which requires more in vitro or in vitro studies.

To sum up, our findings indicated that HUK acts a substantial cardioprotective effect in CME-caused myocardial injury. This protective effect may be attributed to activating the PI3K/Akt/FoxO1 signaling pathway, consequently inhibiting myocardial inflammation and apoptosis significantly. Therefore, we speculate that HUK has promising clinical application potential for pretreating and treating CME-caused myocardial injury and our study will play a certain reference role for the further research of HUK and guide clinical decision-making.

\section{Declarations}

\section{Author Contribution}

Lang Li and Jian Xie designed this study and participated in the revision of the manuscript. Yunhua Lin Guoqing Liu and Qingqing Nong completed the first draft. Bingling Wu, Yuqian Xie and Tao Li were 
mainly responsible for the development of animal experiments.

\section{Acknowledgments}

This work was supported by the First Affiliated Hospital of Guangxi Medical University

\section{Data Sharing Statement}

The data used to support the findings of this study are available from the corresponding author upon request.

\section{Funding}

This work was supported by the National Natural Science Foundation of China (Grant No. 81770346) and the Project for Innovative Research Team in Guangxi Natural Science Foundation (Grant No. 2018GXNSFGA281006).

\section{Disclosure}

The authors declare that they have no conflicts of interest for this work.

\section{References}

1. A. Chen, Z. Chen, Y. Xia, et al. Proteomics Analysis of Myocardial Tissues in a Mouse Model of Coronary Microembolization. Front Physiol. 2018; 9: 1318.

2. Q. Su, L. Li, Y.C. Liu, et al. Effect of metoprolol on myocardial apoptosis after coronary microembolization in rats. World J Emerg Med. 2013; 4: 138-143.

3. B. Mo, X. Wu, X. Wang, et al. miR-30e-5p Mitigates Hypoxia-Induced Apoptosis in Human Stem CellDerived Cardiomyocytes by Suppressing Bim. Int J Biol Sci. 2019; 15: 1042-1051.

4. Z.Q. Chen, Y. Zhou, F. Chen, et al. Breviscapine Pretreatment Inhibits Myocardial Inflammation and Apoptosis in Rats After Coronary Microembolization by Activating the PI3K/Akt/GSK-3beta Signaling Pathway. Drug Des Devel Ther. 2021; 15: 843-855.

5. G. Heusch, A. Skyschally, P. Kleinbongard. Coronary microembolization and microvascular dysfunction. Int J Cardiol. 2018; 258: 17-23.

6. Z.Q. Chen, Y. Zhou, J.W. Huang, et al. Puerarin pretreatment attenuates cardiomyocyte apoptosis induced by coronary microembolization in rats by activating the PI3K/Akt/GSK-3beta signaling pathway. Korean J Physiol Pharmacol. 2021; 25: 147-157.

7. Y. Liu, Y. Liu, X. Huang, et al. Protective effects and mechanism of curcumin on myocardial injury induced by coronary microembolization. J Cell Biochem. 2019; 120: 5695-5703.

8. J.Y. Wang, H. Chen, X. Su, et al. Atorvastatin Pretreatment Inhibits Myocardial Inflammation and Apoptosis in Swine After Coronary Microembolization. J Cardiovasc Pharmacol Ther. 2017; 22: 189195. 
9. Q. Su, L. Li, J. Zhao, et al. Effects of nicorandil on PI3K/Akt signaling pathway and its anti-apoptotic mechanisms in coronary microembolization in rats. Oncotarget. 2017; 8: 99347-99358.

10. Q. Su, X. Lv, Z. Ye. Ligustrazine Attenuates Myocardial Injury Induced by Coronary Microembolization in Rats by Activating the PI3K/Akt Pathway. Oxid Med Cell Longev. 2019; 2019: 6791457.

11. D. Han, X. Chen, D. Li, et al. Human Urinary Kallidinogenase decreases recurrence risk and promotes good recovery. Brain Behav. 2018; 8: e01033.

12. N. Ma, Z.A. Zhao, N.N. Zhang, et al. Intra-arterial human urinary kallidinogenase alleviates brain injury in rats with permanent middle cerebral artery occlusion through PI3K/AKT/FoxO1 signaling pathway. Brain Res. 2018; 1687: 129-136.

13. Y.H. Sun, Q. Su, L. Li, et al. Expression of p53 in myocardium following coronary microembolization in rats and its significance. J Geriatr Cardiol. 2017; 14: 292-300.

14. O. Bikou, S. Tharakan, K.P. Yamada, et al. A Novel Large Animal Model of Thrombogenic Coronary Microembolization. Front Cardiovasc Med. 2019; 6: 157.

15. L. Han, J. Li, Y. Chen, et al. Human Urinary Kallidinogenase Promotes Angiogenesis and Cerebral Perfusion in Experimental Stroke. PLoS One. 2015; 10: e0134543.

16. W. Lan, F. Yang, Z. Li, et al. Human urine kininogenase attenuates balloon-induced intimal hyperplasia in rabbit carotid artery through transforming growth factor beta1/Smad2/3 signaling pathway. J Vasc Surg. 2016; 64: 1074-1083.

17. Z.B. Chen, D.Q. Huang, F.N. Niu, et al. Human urinary kallidinogenase suppresses cerebral inflammation in experimental stroke and downregulates nuclear factor-kappaB. J Cereb Blood Flow Metab. 2010; 30: 1356-1365.

18. A. Dhanasekaran, S.K. Gruenloh, J.N. Buonaccorsi, et al. Multiple antiapoptotic targets of the $\mathrm{PI} 3 \mathrm{~K} /$ Akt survival pathway are activated by epoxyeicosatrienoic acids to protect cardiomyocytes from hypoxia/anoxia. Am J Physiol Heart Circ Physiol. 2008; 294: H724-H735.

19. B. Ebner, S.A. Lange, D. Hollenbach, et al. In situ postconditioning with neuregulin-1 $\beta$ is mediated by a PI3K/Akt-dependent pathway. Can J Cardiol. 2015; 31: 76-83.

20. R. Cortés-Vieyra, A. Bravo-Patiño, J.J. Valdez-Alarcón, et al. Role of glycogen synthase kinase-3 beta in the inflammatory response caused by bacterial pathogens. J Inflamm (Lond). 2012; 9: 23.

21. W. Qin, L. Cao, I.Y. Massey. Role of PI3K/Akt signaling pathway in cardiac fibrosis. Mol Cell Biochem. 2021.

22. F. He, N. Zhang, Y. Lv, et al. Low-dose lipopolysaccharide inhibits neuronal apoptosis induced by cerebral ischemia/reperfusion injury via the PI3K/Akt/FoxO1 signaling pathway in rats. Mol Med Rep. 2019; 19: 1443-1452.

23. D. Kretzschmar, C. Jung, S. Otto, et al. Detection of coronary microembolization by Doppler ultrasound in patients with stable angina pectoris during percutaneous coronary interventions under an adjunctive antithrombotic therapy with abciximab: design and rationale of the High Intensity Transient Signals ReoPro (HITS-RP) study. Cardiovasc Ultrasound. 2012; 10: 21. 
24. Y. Yuan, B. Li, W. Peng, et al. Protective effect of glycyrrhizin on coronary microembolization-induced myocardial dysfunction in rats. Pharmacol Res Perspect. 2021; 9: e00714.

25. Q. Mao, X. Liang, Y. Wu, et al. Resveratrol Attenuates Cardiomyocyte Apoptosis in Rats Induced by Coronary Microembolization Through SIRT1-Mediated Deacetylation of p53. Journal of cardiovascular pharmacology and therapeutics. 2019; 24: 551-558.

26. Q. Su, L. Li, Y. Sun, et al. Effects of the TLR4/Myd88/NF-kB Signaling Pathway on NLRP3 Inflammasome in Coronary Microembolization-Induced Myocardial Injury. Cell Physiol Biochem. 2018; 47: 1497-1508.

27. L. Li, D.-H. Li, N. Qu, et al. The role of ERK1/2 signaling pathway in coronary microembolizationinduced rat myocardial inflammation and injury. Cardiology. 2010; 117: 207-215.

\section{Tables}

Table 1 Sequences of the used primers for RT-qPCR. 


\section{GENE Primer Sequence}

TNF-a Forward: 5'-CGTCGTAGCAAACCACCAAG$3^{\prime}$

Reverse: 5'-GAGGCTGACTTTCTCCTGGT$3^{\prime}$

IL-1 1 Forward: 5'-GGGATGATGACGACCTGCTA3'

Reverse: 5'-TGTCGTTGCTTGTCTCTCCT-

$3^{\prime}$

Bcl-2 Forward: 5'-CCTGGCATCTTCTCCTTCCA$3^{\prime}$

Reverse: 5'-GGACATCTCTGCAAAGTCGC$3^{\prime}$

Bax

Forward: 5'-AAGAAGCTGAGCGAGTGTCT$3^{\prime}$

Reverse: 5'-CCAGTTGAAGTTGCCGTCTG$3^{\prime}$

Caspase- Forward: 5'-TGTCGATGCAGCTAACCTCA3 3'

Reverse: 5'-GCAGTAGTCGCCTCTGAAGA$3^{\prime}$

GAPDH Forward: 5'-TGTGAACGGATTTGGCCGTA$3^{\prime}$

Reverse: 5'-GATGGTGATGGGTTTCCCGT$3^{\prime}$

Abbreviations: RT-qPCR, Real-time quantitative PCR.

\section{Figures}



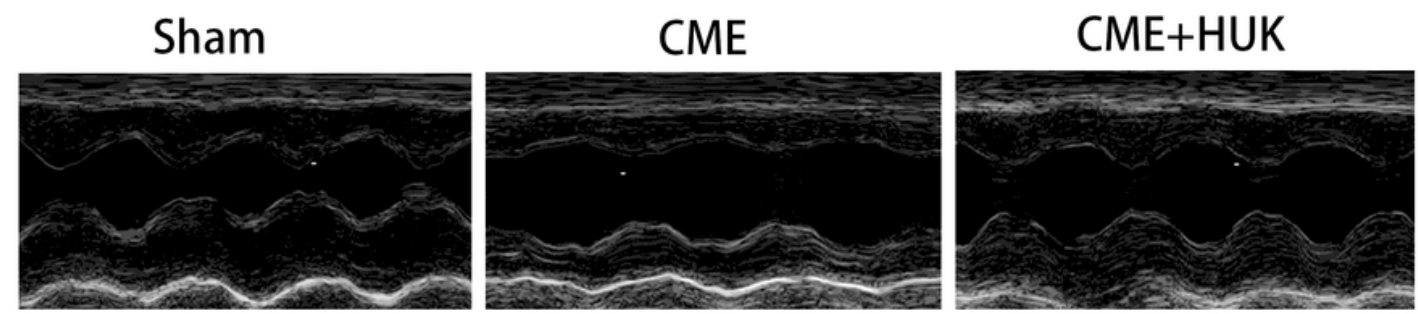

$\mathrm{CME}+\mathrm{HUK}+\mathrm{LY}$
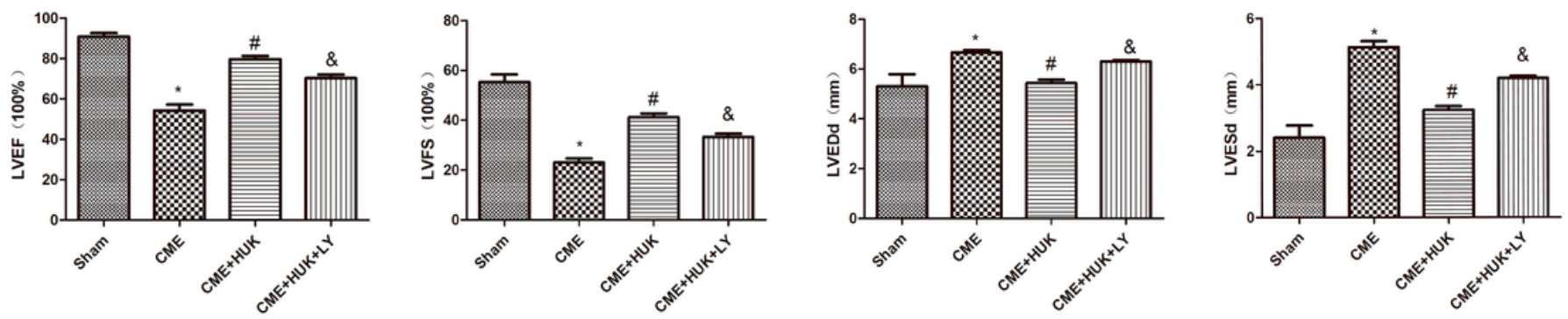

\section{Figure 1}

Cardiac function evaluated by echocardiography in each group ( $n=3$, per group). The cardiac function parameters of left ventricle ejection fraction (LVEF), left ventricle fractional shortening (LVFS), left ventricular end-diastolic diameter (LVEDd), and left ventricular end-systolic diameter (LVESd) were measured quantitatively. Data were presented as the mean \pm standard deviation (SD). ${ }^{*}<0.05$ versus the Sham group; $\# \mathrm{P}<0.05$ versus the CME group; \&P $<0.05$ versus the CME + HUK group. Abbreviations: CME, coronary microembolization; HUK, Human Urinary Kallidinogenase; LY, LY294002. 


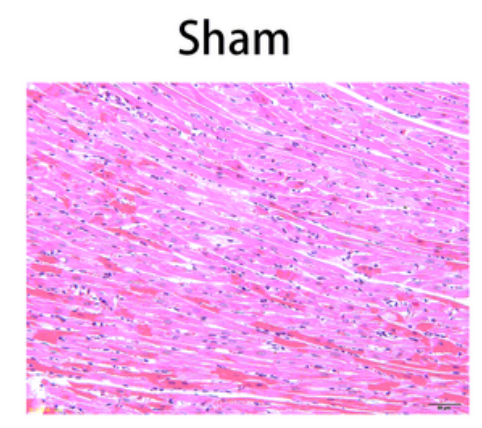

Sham

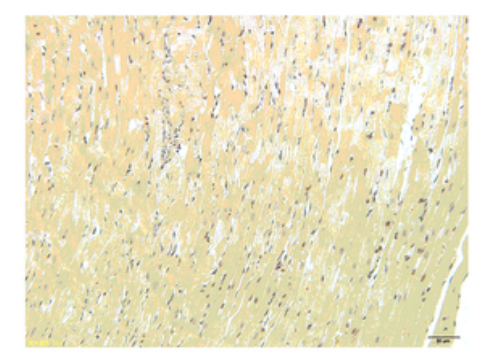

B

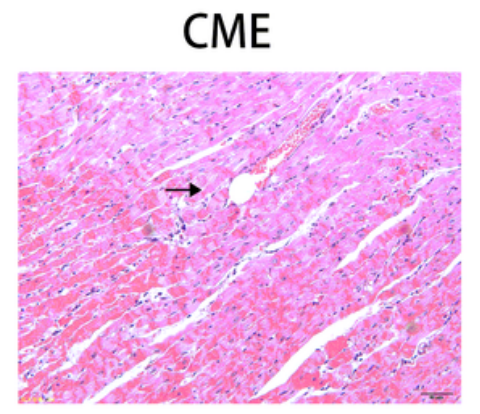

CME

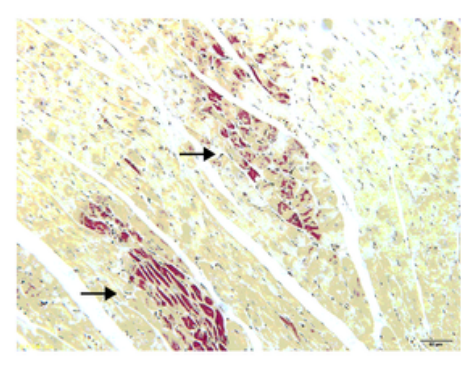

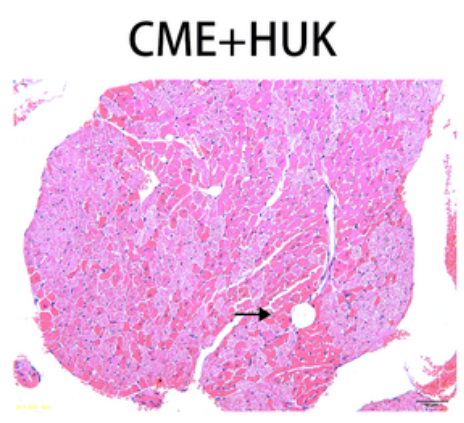

CME+HUK

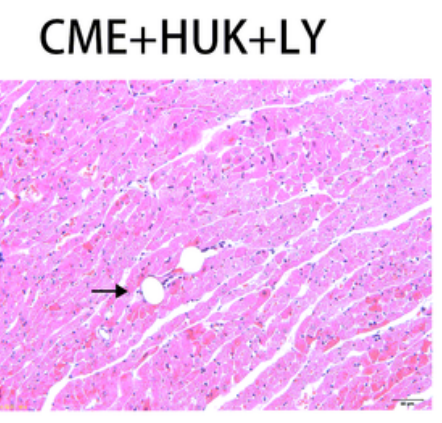

$\mathrm{CME}+\mathrm{HUK}+\mathrm{LY}$
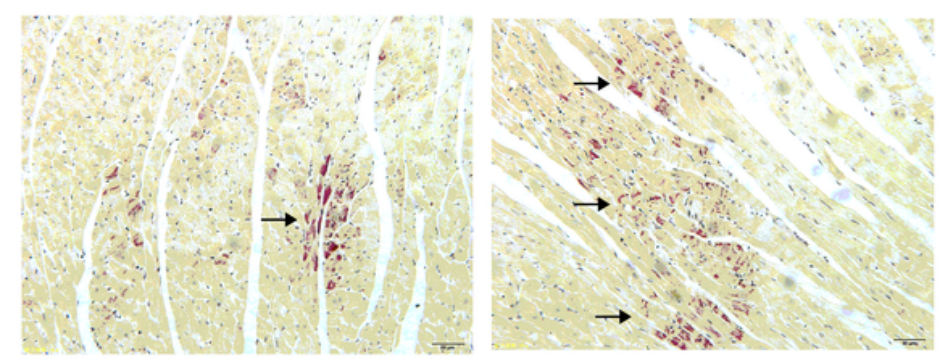

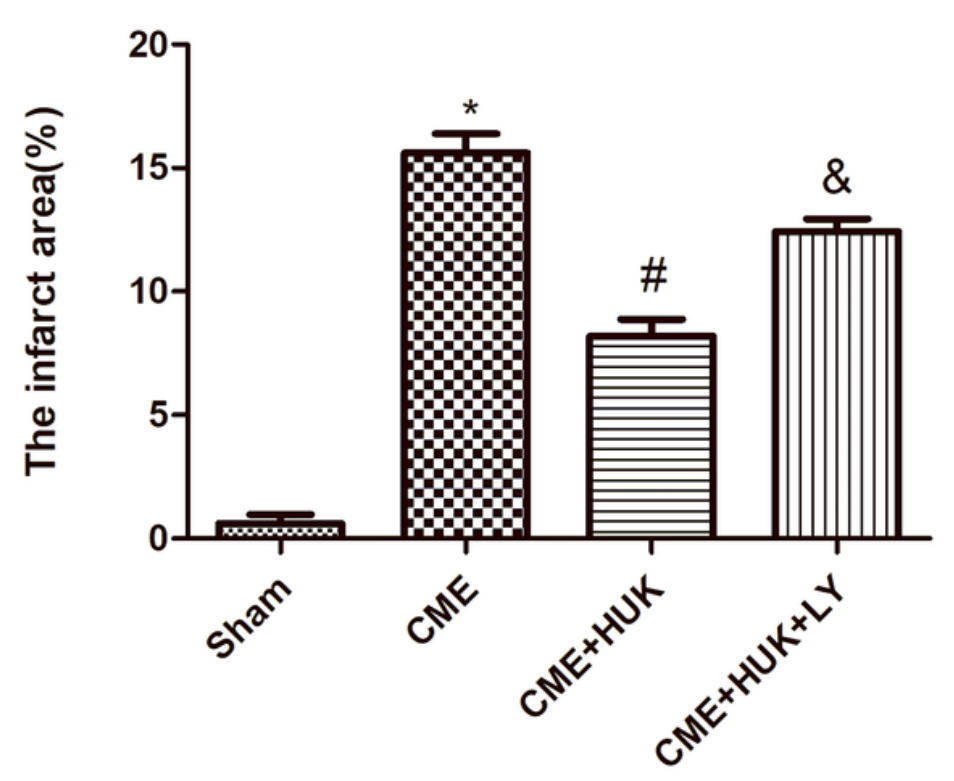

\section{Figure 2}

H\&E and HBFP staining of myocardial tissues ( $\times 200$ magnification; bar $=100 \mu \mathrm{m})(\mathrm{n}=3$, per group). (A) $\mathrm{H} \& \mathrm{E}$ staining. Microspheres were observed in the CME, CME+HUK, and CME + HUK + LY groups but not in the Sham group. The arrows indicated the microspheres. (B) HBFP staining. An ischemic myocardium was highlighted in red. Data were presented as the mean $\pm S D$. ${ }^{*} P<0.05$ versus the Sham group; $\# P<$ 0.05 versus the CME group; \&P $<0.05$ versus the CME + HUK group. Abbreviations: CME, coronary microembolization; HUK, Human Urinary Kallidinogenase; LY, LY294002. 

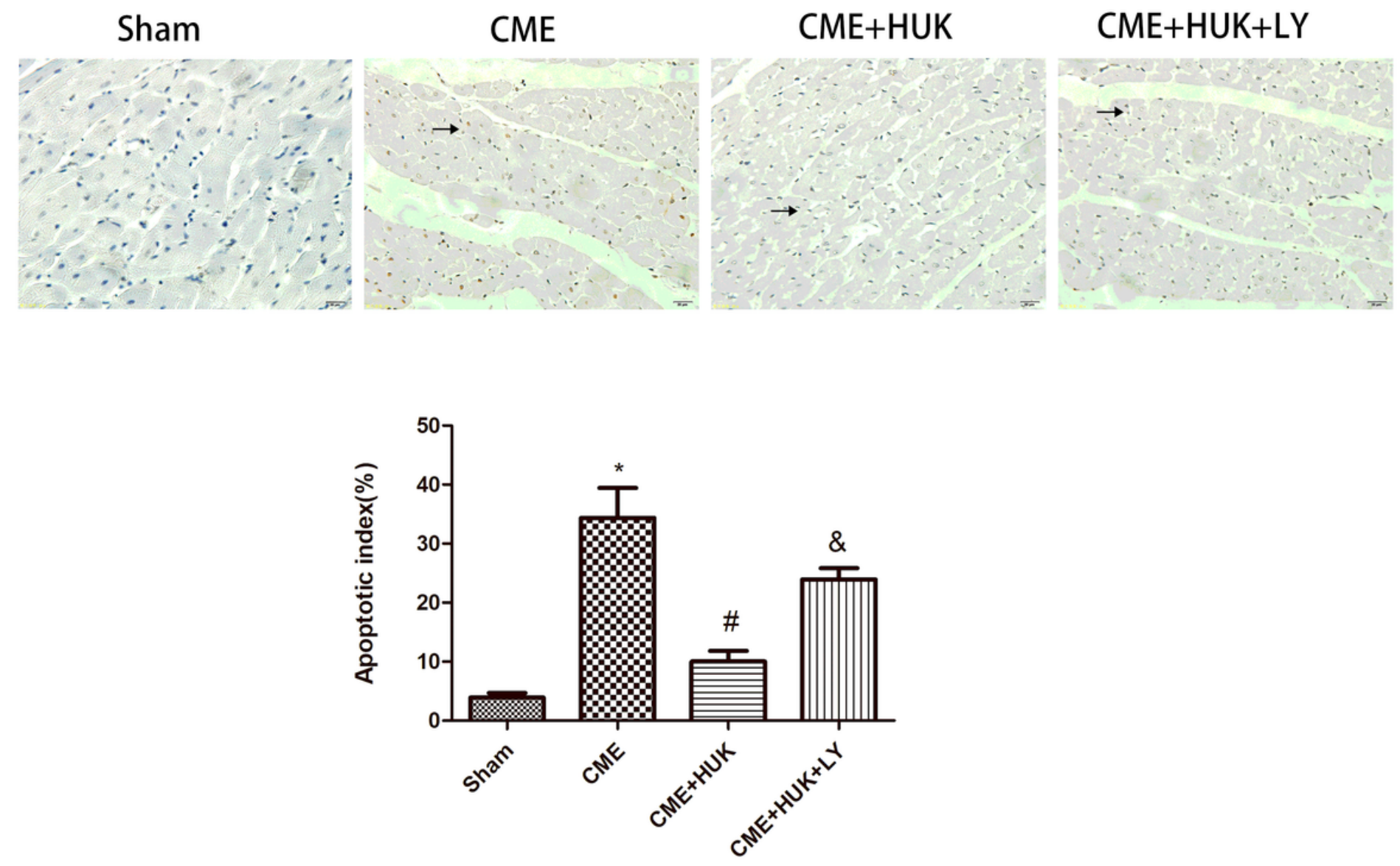

Figure 3

TUNEL staining results of the infarcted and normal cardiomyocyte nuclei and the apoptosis rate of cardiomyocytes in each group $(n=3$, per group). The arrows indicated the microspheres. The nuclei of normal cardiomyocytes were stained light blue, while apoptotic cardiomyocytes' nuclei were stained yellow-brown. Data were presented as the mean $\pm S D$. ${ }^{*} P<0.05$ versus the Sham group; $\# P<0.05$ versus the CME group; \&P < 0.05 versus the CME + HUK group. Abbreviations: CME, coronary microembolization; HUK, Human Urinary Kallidinogenase; LY, LY294002. 
A
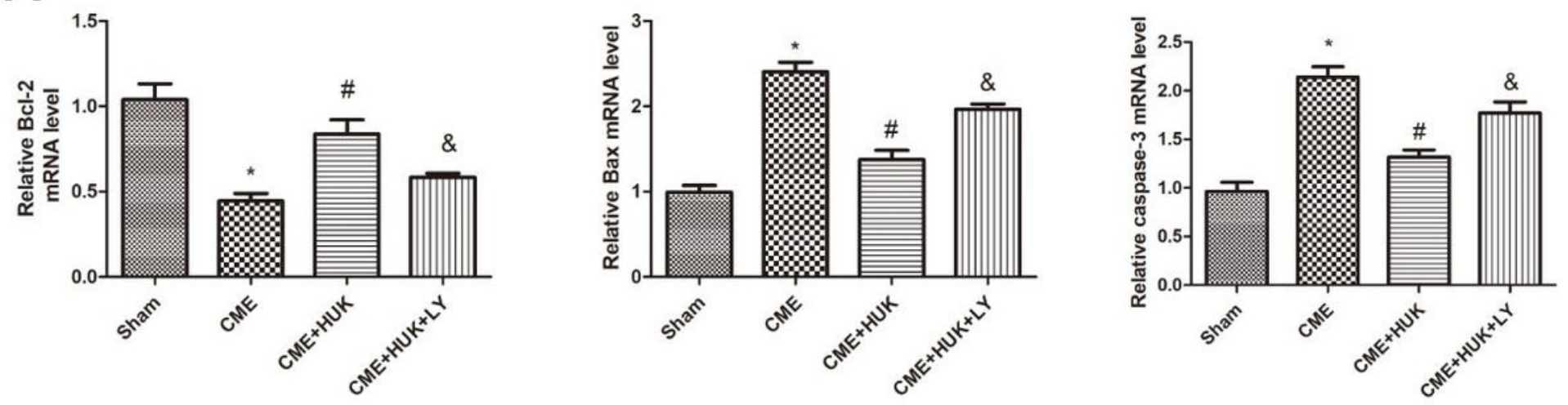

B

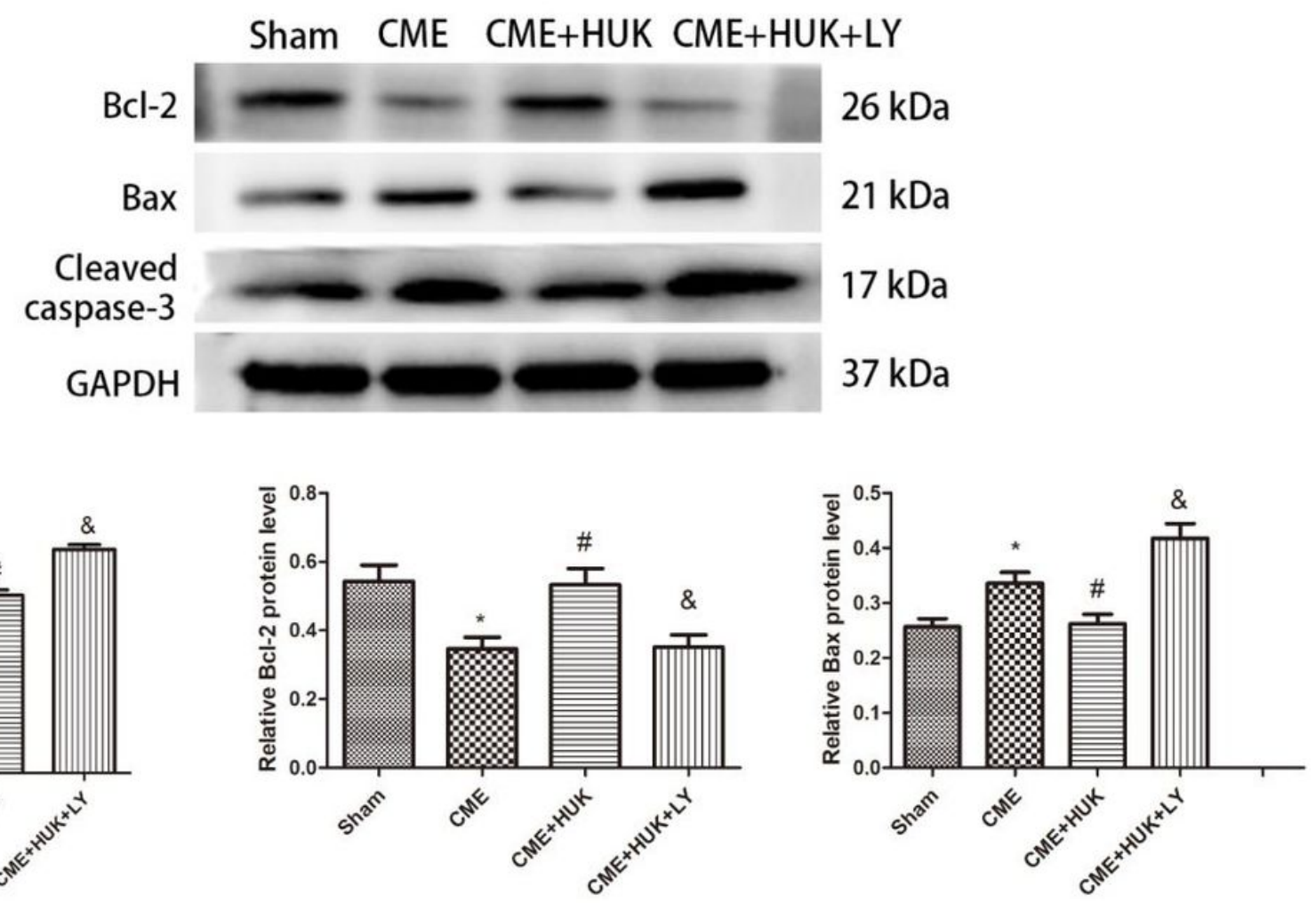

Figure 4

Pretreatment with HUK attenuated CME-induced myocardial apoptosis. (A) The mRNA expression levels of Bcl-2, Bax, and caspase-3 in cardiac tissues ( $n=3$, per group). (B) The protein expression levels of Bcl-2, Bax, and cleaved caspase- 3 in cardiac tissues $(n=3$, per group). Data were presented as the mean $\pm S D$. ${ }^{*} \mathrm{P}<0.05$ versus the Sham group; \#P $<0.05$ versus the CME group; $\& \mathrm{P}<0.05$ versus the CME $+\mathrm{HUK}$ group. Abbreviations: CME, coronary microembolization; HUK, Human Urinary Kallidinogenase; LY, LY294002. 
A

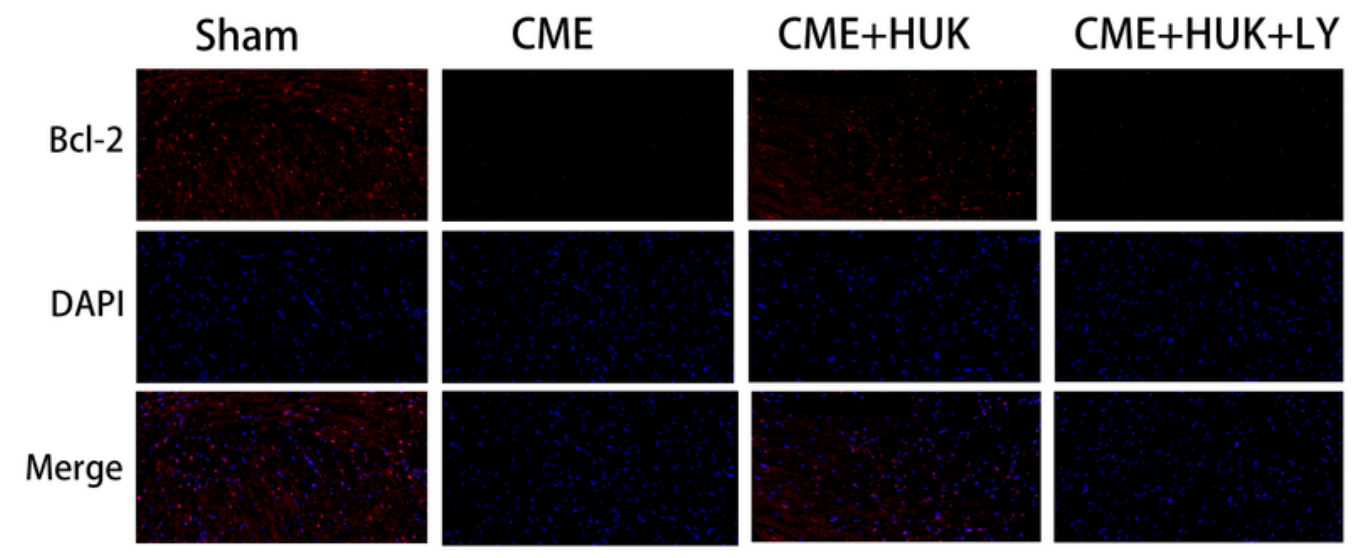

B

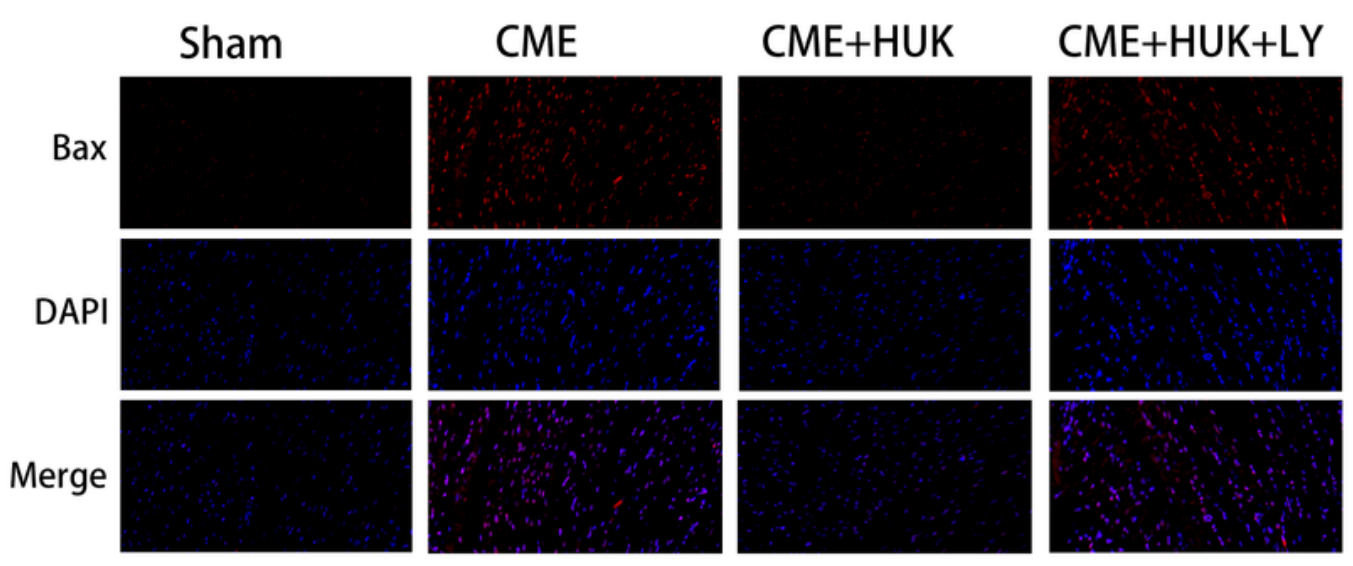

C

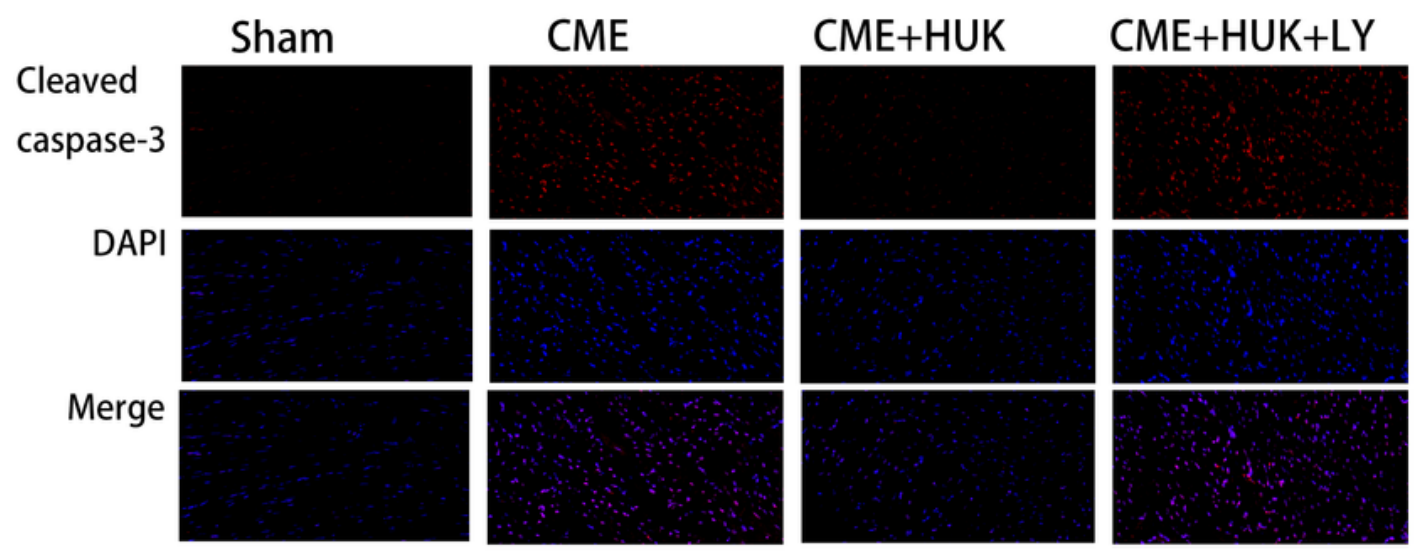

Figure 5

Immunofluorescence staining of Bax, Bcl-2, and cleaved caspase-3 in cardiac tissues. (magnification $\times 400$, scale bar $=25 \mu \mathrm{m})(\mathrm{n}=3$, per group). (A) IF staining detected the expression of Bcl-2. (B) IF staining detected the expression of Bax. (C) IF staining detected the expression of cleaved caspase-3. Abbreviations: CME, coronary microembolization; HUK, Human Urinary Kallidinogenase; LY, LY294002; Immunofluorescence, IF. 
A
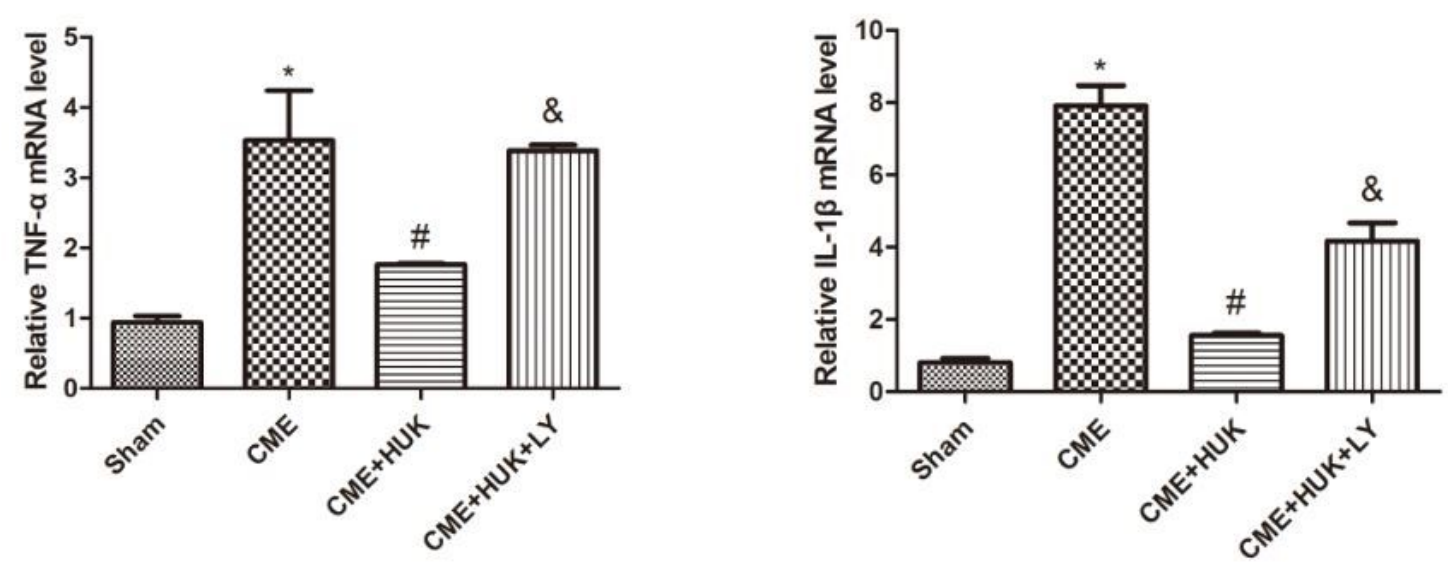

B
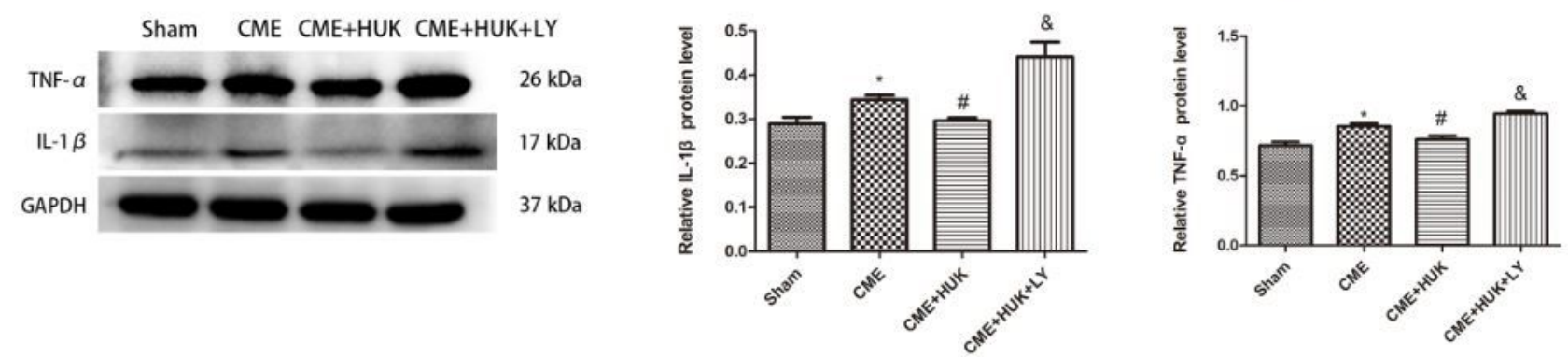

C
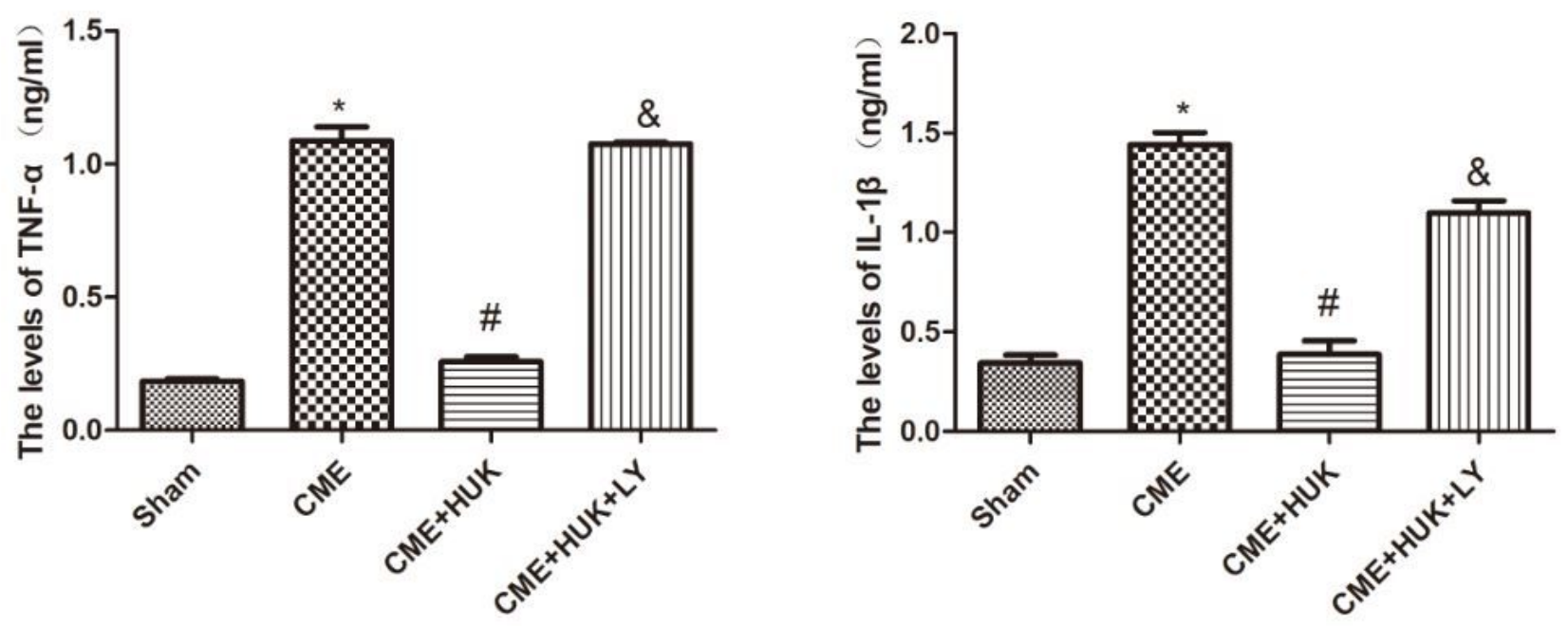

Figure 6

Human Urinary Kallidinogenase reduced the myocardial inflammation $(n=3$, per group). (A) The mRNA expression level of TNF- $\alpha$ and IL-1 $\beta$ in the four groups. (B) The protein expression levels of TNF- $a$ and IL$1 \beta$ in the four groups. (C) The serum levels of TNF- $\alpha$ and IL-1 $\beta$ in the four groups. Data were presented as the mean $\pm S D$. ${ }^{*} P<0.05$ versus the Sham group; $\# P<0.05$ versus the $C M E$ group; $\& P<0.05$ versus the $\mathrm{CME}+\mathrm{HUK}$ group. Abbreviations: CME, coronary microembolization; HUK, Human Urinary Kallidinogenase; LY, LY294002. 
A

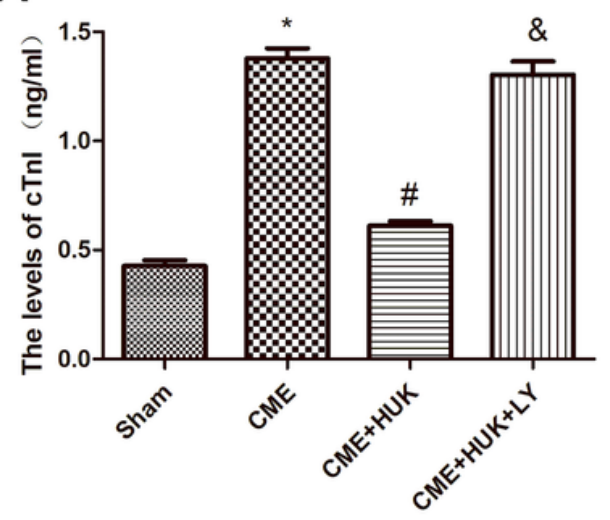

B

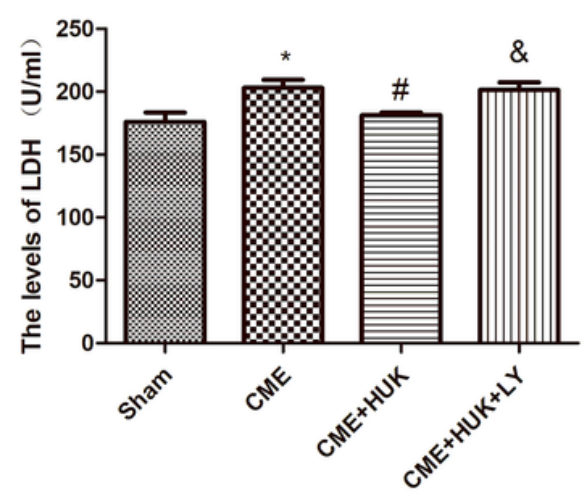

C

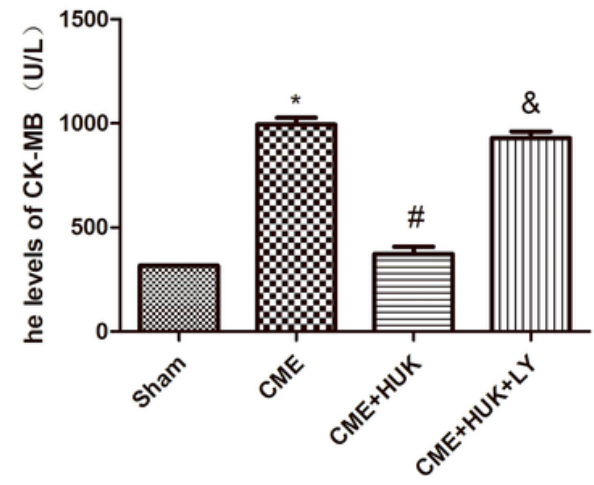

Figure 7

Human Urinary Kallidinogenase reduced the serum levels of myocardial injury markers ( $n=3$, per group). (A) The blood levels of cTnl in the four groups. (B) The blood levels of LDH in the four groups. (C) The blood levels of CK-MB in the four groups. Data were presented as the mean $\pm S D$. ${ }^{*} P<0.05$ versus the Sham group; $\# \mathrm{P}<0.05$ versus the $\mathrm{CME}$ group; \&P $<0.05$ versus the $\mathrm{CME}+\mathrm{HUK}$ group. Abbreviations: $\mathrm{CME}$, coronary microembolization; HUK, Human Urinary Kallidinogenase; LY, LY294002. 

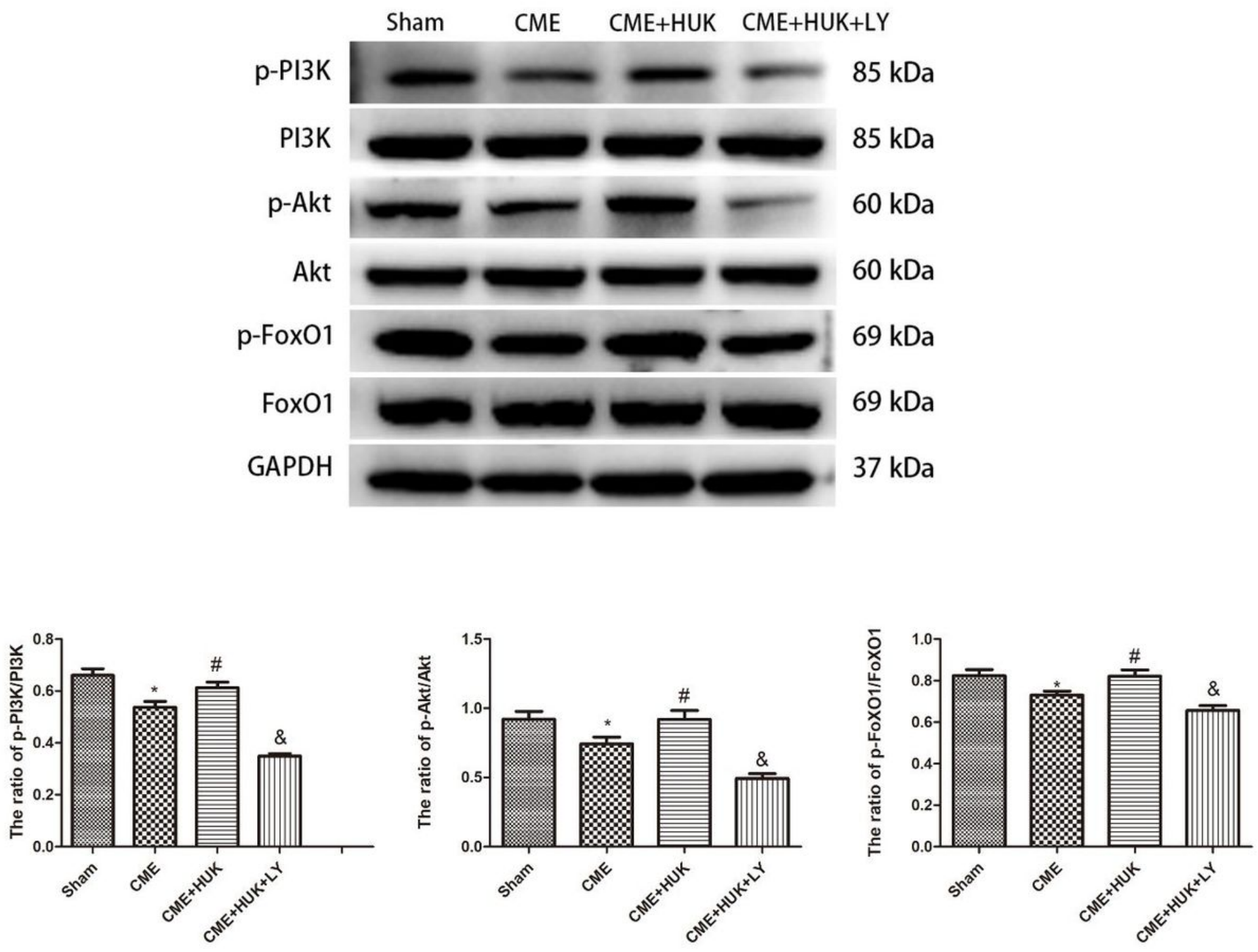

Figure 8

Effect of HUK on PI3K/Akt/Fox01-associated protein expression in cardiac tissues ( $n=3$, per group). Data were presented as the mean $\pm S D$. ${ }^{*} \mathrm{P}<0.05$ versus the Sham group; \#P $<0.05$ versus the CME group; $\& \mathrm{P}<0.05$ versus the $\mathrm{CME}+\mathrm{HUK}$ group. Abbreviations: $\mathrm{CME}$, coronary microembolization; HUK, Human Urinary Kallidinogenase; LY, LY294002. 